\title{
Exercício resistido e hipertrofia regional da musculatura esquelética: revisão de literatura
}

\author{
Anderson Geremias Macedo ${ }^{1}$ \\ David Michel de Oliveira ${ }^{2}$ \\ Astor Reis Simionato ${ }^{3}$ \\ Anderson Bernardino da Silva ${ }^{4}$ \\ Dalton Müller Pessôa Filho ${ }^{5}$
}

Resumo: A hipertrofia do musculo esquelético é caracterizada pelo aumento da área de secção transversal (AST) através de estímulos produzidos pelo exercício resistido (ER). Alguns trabalhos têm demonstrado que os ganhos da hipertrofia muscular esquelética em resposta ao ER ocorrem de forma não uniforme ao longo do músculo, este processo também é conhecido como hipertrofia regional. Este estudo foi constituído por meio de uma revisão bibliográfica. Utilizou-se para coleta a análise de artigos científicos, livros e pesquisas realizadas nas bases de dados do Scielo, LILACS e PubMed, abrangendo trabalhos internacionais. Os trabalhos na literatura são escassos em relação ao ER e hipertrofia regional nos diferentes grupos musculares, o quadríceps parece apresentar maior hipertrofia na região proximal e cadeira extensora nas regiões medial e distal. Já o tríceps a variação das angulações dos exercícios parece influenciar diretamente a hipertrofia regional. Por outro lado o bíceps braquial parece ter maior aumento nas regiões medial e proximal sendo esta resposta influenciada pelo tipo de contração ou velocidade da execução do exercício. Em relação aos isquiotibiais e peitoral maior são insuficientes na literatura os estudos sobre a hipertrofia regional relacionados com estes músculos. Além disso, as diferenças na ativação muscular e a síntese de

1 Pós Doutorando pelo Programa de Pós Graduação em Desenvolvimento Humano e Tecnologias pela Universidade Estadual Paulista Júlio de Mesquita Filho (UNESP), Doutor e Mestre em Ciências Fisiológicas pela Universidade Federal de São Carlos (UFSCAR), Graduado em Educação Física pela Faculdades Associadas de Ensino (UNIFAE). E-mail: andersongmacedo@ yahoo.com.br

${ }^{2}$ Doutor em Alimentos e Nutrição pela Universidade Paulista Júlio de Mesquita Filho (UNESP), Professor Adjunto III da Universidade Federal de Jatai (UFJ). E-mail: profdoliveira@ gmail.com

${ }^{3}$ Mestre em Desenvolvimento Humano e Tecnologias pela Universidade Estadual Paulista Júlio de Mesquita Filho (UNESP), Graduado em Educação Física pela Universidade Estadual Paulista Júlio de Mesquita Filho (UNESP). Docente do Curso de Educação Física da Faculdade Anhanguera de Bauru. E-mail: astor_ars@hotmail.com

${ }^{4}$ Mestre em Saúde da Comunidade pela Universidade de São Paulo (USP), Graduado em Educação Física pela Universidade Estadual Paulista Júlio de Mesquita Filho (UNESP). Docente do Curso de Educação Física da Faculdade Anhanguera de Bauru. E-mail: silva.edfisica@usp.br

${ }^{5}$ Livre-Docente pela Universidade Estadual Paulista Júlio de Mesquita Filho, UNESP, Pós-Doutorado Univversidade Politécnica de Madrid, UPM, Mestre e Doutor em Ciências da Motricidade pela Universidade Estadual Paulista Júlio de Mesquita Filho (UNESP). Graduado em Educação Física pela Universidade Estadual Paulista Júlio de Mesquita Filho (UNESP). Professor Associado no curso de Educação Física da Universidade Estadual Paulista Júlio de Mesquita Filho (UNESP). Docente credenciado no Programa de Pós Graduação em Desenvolvimento Humano e Tecnologias pela Universidade Estadual Paulista Júlio de Mesquita Filho (UNESP). E-mail: dalton.pessoa-filho@unesp.br 


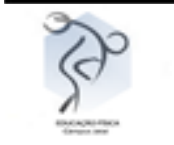

proteínas contráteis parecem ser os principais fatores responsáveis pelas respostas hipertróficas diferentes ao longo do músculo.

Palavras - chave: Hipertrofia regional. Hipertrofia não uniforme. Exercício resistido

\title{
Exercise resistance and regional hypertrophy of skeletal musculature: literature review
}

\begin{abstract}
Skeletal muscle hypertrophy is characterized by increased cross-sectional area (AST) through stimuli produced by resistance exercise (ER). Some studies have shown that gains in skeletal muscle hypertrophy in response to ER occur nonuniformly throughout the muscle, this process is also known as regional hypertrophy. This study was constituted through a bibliographical review. The analysis of scientific papers, books and researches carried out in the SciELO, LILACS and PubMed databases, covering international papers was used to collect data. The work in the literature is scarce in relation to ER and regional hypertrophy in the different muscle groups, the quadriceps seems to present greater hypertrophy in the proximal region and extensor chair in the medial and distal regions. Already the triceps the variation of the angulations of the exercises seems to directly influence the regional hypertrophy. On the other hand, the biceps brachii seems to have greater increase in the medial and proximal regions and this response is influenced by the type of contraction or speed of the execution of the exercise. Regarding the hamstrings and pectoralis major, studies on regional hypertrophy related to these muscles are insufficient in the literature. In addition, differences in muscle activation and contractile protein synthesis appear to be the major factors responsible for different hypertrophic responses throughout the muscle.
\end{abstract}

Keywords: Regional hypertrophy. Nonuniform hypertrophy. Resistance exercise.

\section{INTRODUÇÃO}

A hipertrofia do musculo esquelético é caracterizada pelo aumento da área de secção transversal (AST) através de estímulos produzidos pelo exercício resistido (ER). Este aumento da AST induzido pelo ER ocorre pelo processo de aumento das proteínas contrateis (miosina, actina) e não contráteis (titina, nebulina) (Antonio, 2000; Glass, 2005; Yin,Price, Rudinicki, 2013). Normalmente este hipertrofia é expressa de forma prática pelo aumento anatômico da AST de um músculo ou grupo muscular sendo esta resposta considerada uma adaptação ao treinamento resistido (TR) (Newmire, Willoughby, 2018).

Alguns trabalhos têm demonstrado que os ganhos da hipertrofia muscular esquelética em resposta ao TR ocorrem de forma não uniforme a longo do músculo, este 


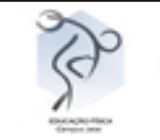

processo também é conhecido como hipertrofia regional (Wakahara et al., 2012; Wakahara et al., 2013; Newmire, Willoughby, 2018). A hipertrofia regional é caracterizada pelo aumento diferenciado da AST nas diferentes áreas do musculo esquelético. Algumas razões para ocorrência da hipertrofia regional são: diferenças na ativação muscular e a síntese protéica dos diferentes tipos de fibras musculares distribuídos ao longo do músculo (Antonio, 2000; Newmire, Willoughby, 2018).

O objetivo desse estudo foi realizar uma discussão, através de uma revisão blibliográfica, sobre a hipertrofia não uniforme induzida pelo TR.

\section{MATERIAIS E MÉTODOS}

Este estudo foi constituído por meio de uma revisão bibliográfica. Utilizou-se para coleta a análise de artigos científicos, livros e pesquisas realizadas nas bases de dados do Scielo, LILACS e PubMed, abrangendo trabalhos internacionais. Para a realização da pesquisa foram utilizados os seguintes termos em português: "hipertrofia muscular esquelética", "hipertrofia regional", "hipertrofia não uniforme", "arquitetura muscular" e ainda as suas combinações. E em inglês: "skeletal muscle hypertrophy "," regional hypertrophy "," nonuniform hypertrophy "," muscular architecture ". Para a confecção do trabalho utilizou-se o critério de inclusão estudos que apresentaram uma relação direta com o tema proposto. A discussão sobre os principais resultados encontrados foram expostos em subtópicos.

\section{EXERCÍCIO RESISTIDO INDUZ A HIPERTROFIA MUSCULAR NÃO UNIFORME AO LONGO DO MÚSCULO}

\subsection{Quadríceps}

O TR pode resultar em uma hipertrofia em vários músculos ou grupos musculares. Krotkiewski et al. (1979) demonstraram inicialmente este fenômeno, neste estudo observou-se 
diferenças do diâmetro muscular em 4 regiões do quadríceps após 5 semanas de treinamento isocinético de extensão de joelho. Narici et al. (1996) mostraram que 6 meses de um protocolo de TR utilizando 3 séries de 8 repetições no leg unilateral houve diferentes aumentos na AST das regiões analisadas dos músculos do quadríceps.

O tipo de contração muscular parece influenciar estas respostas no quadríceps, Seger et al. (1998) mostraram o treinamento excêntrico com a extensão de joelho promoveu maior aumento da AST na região distal do deste músculo. Concordando com estes achados, Martino et al. (2014) verificaram que após 10 semanas de TR utilizando contrações isoladas concêntrica ou excêntrica no leg isocinético, o grupo que realizou apenas a contração concêntrica apresentou maior aumento na AST na região medial do vasto lateral, já o grupo que realizou somente a contração excêntrica este aumento foi maior na região distal. Além disso, a idade ou gênero parece influenciar a hipertrofia regional, Melnyk et al. (2009) examinaram os efeitos do treinamento dinâmico de extensão de joelho nas regiões proximal, medial e distal em 4 grupos [jovens (20-30 anos) e idosos (65-75 anos) do sexos masculino e feminino)]. Os homens jovens e idosos e as mulheres idosas tiveram aumento da AST em todas as regiões do quadríceps, já as mulheres jovens este aumento foi apenas nas regiões medial e distal. Estes resultados indicam que a variação da hipertrofia regional pode depender da idade ou gênero. Em relação ao tipo de exercício, Ema et al. (2013) observaram que indivíduos que participaram do protocolo de 10 semanas de cadeira extensora a região medial e distal do reto foram as regiões que tiveram maior aumento. Já Bloonquist et al. (2013) mostraram que após 12 semanas de TR com agachamento ocorreu maior aumento na região proximal da face anterior da coxa, entretanto não foi feito análises estatísticas entre as regiões. Portanto exercícios envolvendo a extensão de joelho sentado parecem promover maior hipertrofia nas regiões medial e distal dos músculos do quadríceps principalmente reto femoral e vasto lateral. Em relação a exercícios como agachamento ou leg press ainda existe a necessidade de novas pesquisas. A amplitude do movimento também é importante para o desenvolvimento da AST nas diferentes regiões do quadríceps, visto que após 12 semanas de 


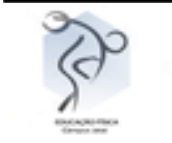

agachamento com amplitudes total (120 graus de flexão de joelho) houve aumento da AST em toda face anterior da coxa, porém o agachamento com amplitude reduzida (60 graus flexão do joelho) notou-se aumento em apenas algumas regiões da face anterior da coxa (Bloonquist et al.,2013).

\section{a. Isquiotibiais}

$\mathrm{Na}$ literatura são escassos os estudos avaliando mudanças regionais da AST induzida pelo TR nos isquiotibiais. Hudelmaier et al. (2010) estudaram as mudanças na AST nos isquiotibiais induzidas pelo TR em mulheres de meia idade envolvendo exercício resistido e endurance. Avalição da AST ao longo do isquiotibiais constitui-se com intervalo de imagens de $10 \%$ sendo mensurado de $10 \%$ a $90 \%$ (proximal para distal), foi observado aumento da área da AST somente nas regiões entre 20\% a 60\%. Já Bloomquist et al. (2013) comparam o efeito de do agachamento curto (60 graus flexão do joelho) e profundo (120 graus de flexão de joelho) na AST da face posterior da coxa de homens jovens. Ambos os grupos mostraram aumento da AST da face posterior somente na segunda região mais proximal dentre as seis regiões avaliada, entretanto somente o grupo que realizou o agachamento profundo apresentou aumento significativo.

\section{b. Tríceps braquial}

Em relação ao tríceps braquial existe um número maior de estudos na literatura analisando a hipertrofia regional neste músculo. Kanesika et al. (2002) observaram significante aumento AST da região medial do trícpes braquial, porém não nas regiões proximal e distal, após o treinamento dinâmico. Já, Housh et al. 1992 encontraram um aumento significante da AST na região medial, após o treinamento isocinético de extensão de cotovelo. O estudo de Wakahara et al. (2012) investigaram mudanças na AST em 13 regiões ao longo do tríceps braquial após 12 semanas de TR envolvendo a extensão de cotovelo deitada, houve maior aumento nas regiões proximal e medial comparado a região distal. Outro 


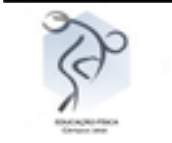

estudo do mesmo autor verificou diferentes das regiões do tríceps braquial utilizando a extensão de cotovelo com halteres "supino fechado" ocorrendo maior aumento da AST na região medial e parte da região distal após o TR (Wakahara et al., 2013). Por conta dos sujeitos estudados serem similares e o programa de TR ser o mesmo, exceto a modalidade de exercício serem diferentes, monoarticular extensão de tríceps deitado "tríceps testa" (Wakahara et al., 2012) vs. multiarticular extensão de tríceps com halter "supino fechado" (Wakahara et al., 2013), os resultados indicam a modalidade ou tipo de exercício pode promover efeitos distintos na hipertrofia ao longo do tríceps braquial.

\section{c. Bíceps braquial}

Já o músculo bíceps braquial ainda não existem muitos trabalhos analisando as mudanças da AST nas diferentes regiões deste músculo. Matta et al. (2011) avaliaram o diâmetro muscular dos bíceps braquial em 3 regiões distintas em indivíduos jovens do sexo masculino após 12 semanas de TR envolvendo diferentes exercícios, houve aumento do diâmetro muscular nas 3 regiões, porém este aumento foi maior nas regiões medial e proximal. Fathing e Chilibek (2003) analisaram tipo e velocidade de contração em 8 semanas de treinamento isocinético com flexão de cotovelo, ao final observou-se maior aumento da AST das três regiões dos flexores do cotovelo na contração excêntrica comparado a concêntrica, além disso a contração excêntrica executada de forma rápida apresentou maiores aumentos nas regiões medial e distal quando comparado a lenta.

\section{d. Peitoral maior}

Até o momento só existe um estudo sobre o peitoral maior. Ogasawara et al. (2013) analisaram os ganhos da AST músculo peitoral maior em homens jovens após 24 semanas de treinamento contínuo ou periódico com supino reto. Houve aumento da AST em todas as regiões analisadas, porém não foi apresentado diferenças entre as regiões. 


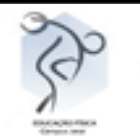

\section{POSSÍVEIS FATORES ENVOLVIDOS NA HIPERTROFIA MUSCULAR NÃO UNIFORME}

\subsection{Ativação muscular}

Tem sido proposto que as diferenças na ativação muscular e a síntese de proteínas contráteis são as possíveis causas das diferentes respostas hipertróficas ao longo do musculo (Narici et al. 1996). Tem sido mostrado que durante a sessão de treinamento a ativação muscular poder ser diferente dentre as regiões deste músculo (Narici et al. 1996; Wakahara et al., 2012, Wakahara et al., 2013). O músculo esquelético não é apenas um conjunto de fibras musculares que interage apenas com um único nervo. Em vez disso, ele pode ser dividido em compartimentos neuromusculares que são regiões distintas deste músculo, a qual cada uma é inervada por um ramo nervoso individual (Antônio, 2000). Embora as evidências para os compartimentos neuromusculares em humanos sejam limitadas para muitos músculos, estes compartimentos parecem explicar as diferenças da ativação regional durante uma sessão de treino. Os estudos de Wakahara et al., (2012) e (2013) usando o imagens de ressonância magnética como um índice de ativação muscular mostraram essas diferenças na ativação do músculo tríceps braquial. Wakahara et al (2012) observaram maior ativação muscular das regiões proximal e medial comparadas a região distal, durante a realização do exercício extensão de cotovelo deitado tríceps deitado "tríceps testa". O mesmo autor utilizando a mesma técnica Wakahara et al (2013) mostraram que a ativação das regiões medial e distal foram maiores compradas a região proximal durante a realização da extensão de cotovelo com halteres "supino fechado". Estes resultados sugerem que a hipertrofia muscular, em partes pode ser causada pela pelas diferenças regionais na ativação muscular durante a execução do exercício.

\subsection{Síntese de proteínas}


A síntese de proteínas aumenta em função da sobrecarga mecânica imposta pelo TR e esta resposta pode depender dos tipos de fibras musculares (D'Antona et al., 2006). Nos músculos de humanos, as mudanças hipertróficas na AST da fibra musculares induzidas pelo TR diferem entre tipo I e tipo II (Aagaard et al., 2001). Por conta das diferenças proporções das fibras do tipo I e II ao longo do músculo, o tipo de fibra muscular pode desempenhar um papel importante na hipertrofia não uniforme no músculo esquelético (Aagaard et al., 2001). Considerando a composição do tipo de fibra muscular do músculo, Elder et al. (1982) encontraram vários tipos de fibras musculares ao longo do músculo vasto lateral. Além disso, (LEXELL,HENRIKSSON-LARSEN, SJOSTROM, 1983) demonstraram que as fibras do tipo I são mais profundas no musculo vasto lateral, enquanto as fibras do tipo II são predominantemente mais superficiais. A distribuição das fibras musculares ao longo do fascículo do músculo vasto lateral do tipo II encontra-se mais perifericamente e são uniformemente pouco distribuídas (Pernus, Erzen, 1991). Além disso, as fibras do tipo II também são distribuídas mais perifericamente no fascículo dos músculos bíceps braquial, deltoide e quadríceps (Manta et al., 1996). O latíssimo do dorso apresenta maior quantidade de fibras de contração rápida nos segmentos anterolateral e medial em comparação ao segmento superior (Sola et al., 1990). Desta maneira, as diferenças regionais da composição dos tipos de fibras musculares de um músculo podem ocasionar repostas distintas ao longo deste músculo. Consequentemente, a diferença da hipertrofia regional em um músculo pode estar relacionada com a síntese proteica dos diferentes tipos de fibras musculares encontradas nas diferentes regiões.

\section{CONSIDERAÇÕES FINAIS}

Ainda são escassos os estudos na literatura sobre a hipertrofia não uniforme do músculo esquelético. Porém a hipertrofia regional no musculo induzida pelo TR parece estar envolvida principalmente pela ativação muscular das diferentes regiões e pela síntese de proteínas dos diferentes tipos de fibras musculares distribuídas ao longo deste músculo. Em 


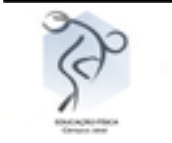

termos práticos, a variação de exercícios é fundamental para hipertrofia uniforme de um músculo. Pois o fato do músculo responder de maneira não uniforme indica que existem diferentes padrões de recrutamento que podem ser alcançados através de diferentes tipos de exercícios resistidos e isto promoverá a hipertrofia de maneira uniforme nas diferentes regiões do músculo.

\section{REFERÊNCIAS}

AAGAARD, P., ANDERSEN, J. L., DYHRE-POULSEN, P., LEFFERS, A. M., WAGNER, A., MAGNUSSON, S. P., HALKJAER-KRISTENSEN, J., SIMONSEN, E. B. A mechanism for increased contractile strength of human pennate muscle in response to strength training: changes in muscle architecture. The Journal of Physiology. Vol. 534. Num.15. 2001. p. 613-623.

BLOOMQUIST, K., LANGBERG, H., KARLSEN, S., MADSGAARD, S., BOESEN, M., RAASTAD, T. Effect of range of motion in heavy load squatting on muscle and tendon adaptations. European Journal of Applied Physiology. Vol. 113. Num. 8. 2013. p. 21332142.

D'ANTONA, G., LANFRANCONI, F., PELLEGRINO, M. A., BROCCA, L., ADAMI, R., ROSSI， R., MORO, G., MIOTTI， D., CANEPARI， M., BOTTINELLI， R. Skeletal muscle hypertrophy, structure, and function of skeletal muscle fibres in male body builders. The Journal of Physiology. Vol. 570. Num. 3. 2006. p. 611-627.

ELDER, G. C., BRADBURY, K., ROBERTS, R. Variability of fiber type distributions within human muscles. J Appl Physiol Respir Environ Exerc Physiol. Vol. 53. Num. 06. 1982. p. 1473-1480.

HOUSH, D.J., HOUSH T. J., JOHNSON, G. O., CHU, W. K. Hypertrophic response to unilateral concentric isokinetic resistance training. Journal of Applied Physiology Vol. 73. Num. 1. 1992. p. 65-70.

HUDELMAIER, M., WIRTH, W., HIMMER, M., RING-DIMITRIOU, S., SÄNGER, A., ECKSTEIN, F. Effect of exercise intervention on thigh muscle volume and anatomical cross-sectional areas--quantitative assessment using MRI. Magnetic Resonance in Medicine. Vol. 64. Num. 6. 2010. p. 1713-1720. 


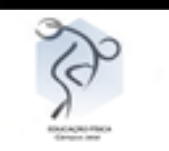

JOSE, A. Nonuniform Response of Skeletal Muscle to Heavy Resistance Training: Can Bodybuilders Induce Regional Muscle Hypertrophy? Journal of Strength and Conditioning Research. Vol. 14. Num. 1. 2000. p. 102-113.

KROTKIEWSKI, M., ANIANSSON, A., GRIMBY, G., BJÖRNTORP, P., SJÖSTRÖM, L. The effect of unilateral isokinetic strength training on local adipose and muscle tissue morphology, thickness, and enzymes. European Journal of applied Physiology and occupational Physiology. Vol. 42 . Num. 4. 1979. p. 271-281.

LEXELL, J., HENRIKSSON-LARSEN, K., SJOSTROM, M. Distribution of different fiber types in human skeletal muscles. 2. A study of cross-sections of whole m. vastus lateralis. Acta Physiologica. Scandinavica. Vol. 117. Num. 1. 1983. p.115-122.

MANTA, P., KALFAK, N., KARARIZOU, E., VASSILOPOULOS, D., PAPAGEORGIOU, C. Size and proportion of fiber types in human muscle fascicles. Clinical Neuropathology. Vol. 15. Num. 2. 1996. p. 116-118.

MATTA, T., SIMÃO, R., DE SALlES, B. F., SPINETI, J., OLIVEIRA, L. F. Strength training's chronic effects on muscle architecture parameters of different arm sites. Journal of Strength and Conditioning Research. Vol. 25. Num. 6. 2011. p. 1711-1717.

MELNYK, J. A., ROGERS, M. A., HURLEY, B. F. Efects of strength training and detraining on regional muscle in young and older men and women. European Journal Applied Physiology. Vol. 105. Num. 06. 2009. p. 929-938.

MIYAMOTO, N., WAKAHARA, T., EMA, R., KAWAKAMI, Y. Non-uniform muscle oxygenation despite uniform neuromuscular activity within the vastus lateralis during fatiguing heavy resistance exercise. Clinical Physiology and functional imaging. Vol. 33. Num. 6. 2013. p. 463-469.

OGASAWARA, R., YASUDA, T., ISHII, N., ABE T.Comparison of muscle hypertrophy following 6-month of continuous and periodic strength training. European Journal of Applied Physiology. Vol. 104. Num. 13. 2013. p. 975-985.

PERNUS, F., ERZEN, I. Arrangement of fiber types within fascicles of human vastus lateralis muscle. Muscle \& Nerve. Vol. 14. Num. 4. 1991. p. 304- 309.

SEGER, J. Y., ARVIDSSON, B., THORSTENSSON, A. Specic effects of eccentric and concentric training on muscle strength and morphology in humans. European Jounal of Applied Physiology. Vol. 79. Num. 1. 1998. p. 45-57. 
SOLA, O.M., HAINES, L.C. KAKULAS, B.A. IVEY, T. D. DILLARD, H. THOMAS, R. SHOJI, Y. FUJIMURA, Y. DAHM. L. Comparative anatomy and histochemistry of human and canine latissimus dorsi muscle. The Journal of Heart Transplantation. Vol. 9. Num. 2. 1990. p. 151-159.

WAKAHARA, T., MIYAMOTO, N., SUGISAKI, N., MURATA, K., KANEHISA, H Association between regional differences in muscle activation in one session of resistance exercise and in muscle hypertrophy after resistance training. European Journal of Applied Physiology. Vol. 112. Num. 4. 2012. p. 1569-76.

YIN, H. PRICE, F., RUDNICKI, M. A. Satellite cells and the muscle stem cell niche. Physiolgical Review. Ottawa. Vol. 93. Num. 1. 2013. p. 23-67. 